\title{
Modelling time efficiency of cobot-supported kit preparation
}

\author{
Patrik Fager $^{1} \cdot$ Martina Calzavara $^{2} \cdot$ Fabio Sgarbossa $^{2,3}$
}

Received: 25 August 2019 / Accepted: 10 November 2019 / Published online: 18 December 2019

(C) The Author(s) 2019

\begin{abstract}
Kitting - meaning to supply assembly with components in presorted kits - is widely seen as beneficial for assembly quality and efficiency when there is a multitude of component variants. However, the process by which kits are prepared - the kit preparation - is labour-intensive, and kit errors are problematic at assembly processes. The use of robotics to support kit preparation has received some attention by researchers, but literature is lacking with respect to how collaborative robots - cobots - can support kit preparation activities. The purpose of this paper is to identify the potential of a cobot to support time-efficient batch preparation of kits. To address the purpose, the paper presents a mathematical model for estimation of the cycle time associated with cobotsupported kit preparation. The model is applied in a numerical example with experimental data from laboratory experiments, and cobot-supported kit preparation is compared with manual kit preparation. The findings suggest that cobot-supported kit preparation is beneficial with diverse kits and smaller components quantities per SKU (Stock Keeping Unit) and provides less variability of the outcome, when compared to manual kit preparation. The paper reveals several insights about cobotsupported kit preparation that can be valuable for both academics and practitioners. The model developed can be used by practitioners to assess the potential of cobots to support kit-batch preparation in association with assembly, spare parts, repair and maintenance, or business to business industry.
\end{abstract}

Keywords Kitting $\cdot$ Robotics $\cdot$ Mixed-model assembly $\cdot$ Collaborative robots $\cdot$ Order picking $\cdot$ Mathematical modelling

\section{Introduction and aim of the study}

Mixed-model assembly often involves a multitude of component variants and effective materials supply is essential. Kitting is here a frequently adopted materials supply principle that involves supplying assembly with components in presorted kits [1], widely seen as beneficial when there is large variety of components $[2,3]$. With kitting, the process by which kits are prepared - the kit preparation - is typically reported as labour-intensive, and time efficiency is key for keeping running costs low [4]. Moreover, manual kit prepara-

Patrik Fager

fagerp@chalmers.se

1 Department of Technology Management and Economics, Chalmers University of Technology, Gothenburg, Sweden

2 Department of Management and Engineering, University of Padua, Padua, Italy

3 Department of Mechanical and Industrial Engineering, Norges Teknisk-Naturvitenskapelige Universitet, Trondheim, Norway tion is prone to human errors that lead to errors in kits, which are disruptive and costly at assembly processes $[5,6]$. Recent research has shown that robots can support time-efficient kit preparation by relieving pickers of some of the tasks in kit preparation while simultaneously supporting kit quality by removing the risk of human errors [7-9]. However, kit preparation supported by cobots - that is, collaborative robots that share workspaces with pickers - has so far received less attention, and there is considerable confusion in industry with regard to how cobots can be used in kit preparation.

The purpose of this paper is to identify the potential of a cobot to support time-efficient batch preparation of kits. To address the purpose, the paper presents a mathematical model for estimating the cycle time of cobot-supported kit preparation, useful also for understanding how the operator's time allocation among tasks change when a cobot is introduced. The considered cobot-application consists of a cobot mounted on an AGV that sorts components that have been picked by an operator into a batch of kits. An operator and a cobot collaborate throughout the work cycle, and the cycle time of the process depends on how well the collaboration works. The application was already presented in Fager et al. [10], showing 
that cobot-supported batch preparation of kits results in comparable cycle time as manual preparation, but with a more stable cycle time. This paper advances the analysis, by taking into account typical production system characteristics, including kit commonality (unique or identical contents in different kits) and the average number of components picked per part number, which are important production systems characteristics with respect to kit preparation [2]. The model also considers the number of compartments in the shared workspace between the operator and the cobot - in which the operator can drop components associated with a single part number which is considered as a decision variable, since it affects how the collaboration works. The model is applied with experimental data to compare cobot-supported kit preparation with manual kit preparation. The paper contributes to the existing knowledge by presenting a model that gives a detailed view of how time efficiency as associated with batch preparation of kits is affected when using a cobot to support component sorting. This is important because full automation of industrial tasks is often the focus in the literature, while collaborative applications are rare.

The remainder of the paper is structured as follows. In the next section, previous research that has dealt with robotics related to kit preparation is reviewed. Thereafter, the application for cobot-supported kit preparation is described, and assumptions are discussed. After that, the mathematical model for estimating the cycle time is developed, followed by a presentation of results from a numerical example. At the end, the conclusions are presented.

\section{Literature analysis}

The development and the improvement of the technologies used for the design of collaborative robots, together with the reduction of the investment required for their installation, have led to an interesting spread of these systems [11, 12]. Recent research has shown how a proper programming of a cobot can be crucial to guarantee a successful use of such a device [13]; other important aspects are related to the used visual system and to the effectiveness of the grasp action $[14,15]$. For now, most of the investigated applications are related to assembly environments, in which cobots are used to support the operator during the execution of the tasks [16-18]. In this direction, Krüger et al. [19] surveyed human-machine interaction and identified research issues related to collaboration between workers and robots. They highlighted that typical advantages with robots are that they can produce without breaks and fatigue while also providing exceptional productivity for simple assembly tasks. Conversely, human workers provide exceptional sensomotoric abilities for handling complex task, and the key with collaborative applications is to promote the advantages of both parties.
Among all their possible applications, cobots can also be used to support kit preparation, for example, by helping the operator in sorting the items, reducing picking errors, and guaranteeing more stable cycle times [7, 8]. Coelho et al. [8] developed a simulation model and compared various kitting applications, some of which involved cobots. Their results suggest that human workers are associated with higher productivity, but that the cobots are better at dealing with uncertainty and for reducing output variability. Martinez et al. [14] deepened the issue of correct identification and grasp of objects, by developing a vision system for supporting 3D bin picking of randomly oriented items. Their system was capable of recognising the location of a component in the bin to validate that the component was picked up and placed at the right location. Wakabayashi et al. [20] modelled an application of kit preparation with autonomous and collaborative storage shelves, which presented the picker with components in a parts-to-picker fashion. The application showed a reduction in working time, due to decreased walking distances. Caputo et al. [2] modelled three applications of kit preparation with different automation levels: manual kitting, manual kitting supported by a picking information system, and automated retrieval with manual sorting. The model showed that automated retrieval becomes economically beneficial for higher kit production volumes.

Boudella et al. [9] discussed robotic kit preparation and highlighted three main system components: the manipulator (robot arm), the vision system (camera), and the end effector (gripper). In their analysis, they identified challenges with robotic kit preparation, for example, selecting the correct gripper with respect to the component characteristics, and how to handle additional tasks such as removal of empty containers. Boudella et al. [7] modelled robotic bin picking of components for allocating SKUs (Stock Keeping Units) in a hybrid robot-worker cell for kit preparation. The robot and the operator worked in separately and completed different parts of the kits. The model considered how empty containers and inner packing could be handled by the robot, although there was no collaboration between the robot and the worker during the work cycle, and the robot did not sort the components into the kits, placing them on a conveyor.

Sellers and Nof [21] modelled four types of automationsupported applications for kit preparation and made comparisons among these and two typical manual applications. The applications were compared on a basis of average production rate, average time of orders spent within the system, and average robot utilisation rate under variable operating conditions in the production system, including various dispatching rules when kits should be delivered to assembly, kit variety, and order mix. From 88 simulation runs under varying operating conditions, they found that a mini-load system combined with an on-trolley robot arm was the more productive automationsupported application. 
From the above, it can be seen that cobots applied with kit preparation have not received much attention in previous research, Fager et al. [10] and Coelho et al. [8] marking exceptions. It can also be seen that there have been a few reports of robot arms applied for carrying out component picking in kit preparation activities [e.g. 7, 9] and that there are vision systems capable of supporting this task [14]. While literature that considers applications of kit preparation that involve cobots is scant, the available literature suggests there is a potential of better dealing with uncertainty and reduced variability of outputs $[8,10]$. However, no studies exist that can account for the effects of using cobots to support the sorting of components into kit containers when order batching is applied, accounting for production system characteristics and decision variables associated with cobot application.

\section{Modelling of manual and cobot-supported kit preparation}

This section presents descriptions of kit preparation when it is performed manually by an operator and when it is performed by an operator assisted by a cobot. Thereafter, mathematical models for manual kit preparation and cobot-supported kit preparation are presented. Notations for all model parameters used in the model, and example values used in the model application (Sect. 4), are shown in Table 1 and Table 2. The example values are based on experiments data reported in Fager et al. [22] and Fager [23] for manual kit preparation, and the cobots example values are based on values reported in literature [e.g. 7] and observations in a laboratory.

\subsection{Description of manual and cobot-supported kit preparation}

The kit preparation process supplies a mixed-model assembly process with kits of components. The kits are prepared at a kit preparation workspace separated from assembly. Each kit contains a collection of components that are assembled onto one end product. Kits are prepared according to an order batching approach, meaning that several kits are prepared during every work cycle. At assembly, there is no requirement on the orientation and position of the components in the kits. An overview of manual and cobot-supported kit preparation is shown in Fig. 1.

\subsubsection{Manual kit preparation}

When kit preparation is performed manually, an operator is responsible for picking components from storage and sorting these into kits. A picking information system provides information about which components to pick and in which kits to sort them. The picking information system allows the operator to receive instructions and to confirm activities and, thereby, get feedback that components are picked and sorted correctly. The kit containers are carried by an AGV, which moves from shelf to shelf in accordance with the confirmations performed by the operator. The operator receives instructions about which SKU to pick components of, the quantity to pick, which kits to place components in, and how many components to place in each kit. When components of an SKU are picked, the operator performs an SKU confirmation, and, then, information about in which kit containers the components have to be placed is provided. Thereafter, the operator sorts the components into kits, one kit at a time, and confirms a kit after all components have been sorted into it. This is repeated until all components have been sorted into kits.

\subsubsection{Cobot-supported kit preparation}

When kit preparation is performed by an operator assisted by a cobot, the operator picks components from the shelves in the same way as with manual kit preparation. However, after picking, the operator now puts the full quantity of an SKU in a container presented on the AGV - here called the collaborative work zone. The collaborative work zone can be divided into compartments, where the operator can put the full quantity of an SKU in any of the compartments. A cobot, which is mounted on the $\mathrm{AGV}$, then sorts the components in each compartment into kits. A vision system guides the cobot to perform its task correctly, and the camera is mounted above the collaborative work zone and continually analyses the zone's contents so that the cobot never has to wait for the analysis to complete. To be able to grip all component variants, the cobot has different grippers at its disposal. It changes its gripper depending on the component characteristics, by accessing a tool holder positioned at its base.

\subsection{Model of manual kit preparation}

In the following, a model for estimating cycle time of manual kit preparation is presented. An overview of the model notations is shown in Table 1.

In each work cycle, kits are prepared in a batch of $B$ kits. Each kit should hold the same number of components when complete, but the kits can contain different component variants, corresponding to different SKUs. During each work cycle, $N$ different SKUs are kitted, corresponding to $N$ order lines. For each order line $i$, the operator is provided with instructions of which SKU to pick, what quantity $q$, which kits to place components in, and how many components to place in each kit. Manual kit preparation and order picking activities have been modelled before [see, e.g. 24]. The cycle time of manual kit preparation, $T_{M}$, is generally composed of three activities: travelling $\left(T_{O P}^{\text {trav }}\right)$, picking $\left(T_{O P}^{\text {pick }}\right)$, and sorting $\left(T_{O P}^{\text {sort }}\right)$. Accordingly: 
Table 1 Notations and example values used in the paper

\begin{tabular}{|c|c|c|c|}
\hline Notation & Description & Model* & Example values \\
\hline$T_{M}$ & Cycle time (s) of manual kit preparation & M & - \\
\hline$T_{C}$ & Cycle time (s) of cobot-supported kit preparation & $\mathrm{C}$ & - \\
\hline$T_{O P}$ & Time expenditure (s) by the operator & $\mathrm{M} \& \mathrm{C}$ & - \\
\hline$T_{C B}$ & Time expenditure (s) by the cobot & $\mathrm{C}$ & - \\
\hline$T_{O P}^{\text {trav }}$ & Time expenditure (s) by the operator on travelling activities & $\mathrm{M} \& \mathrm{C}$ & - \\
\hline$T_{O P}^{p i c k}$ & Time expenditure (s) by the operator on picking activities & $\mathrm{M} \& \mathrm{C}$ & - \\
\hline$T_{O P}^{\text {sort }}$ & Time expenditure (s) by the operator on sorting activities & M & - \\
\hline$T_{C B}^{\text {trav }}$ & Time expenditure (s) by the cobot on travelling & $\mathrm{C}$ & 0 \\
\hline$T_{C B}^{v i s}$ & Time expenditure (s) by the cobot on vision system analysis & $\mathrm{C}$ & 0 \\
\hline$T_{C B}^{\text {tool }}$ & Time expenditure (s) by the cobot on tool change & $\mathrm{C}$ & - \\
\hline$T_{C B}^{g r i p}$ & Time expenditure (s) by the cobot on gripping components & $\mathrm{C}$ & - \\
\hline$T_{C B}^{\text {sort }}$ & Time expenditure (s) by the cobot on sorting components & $\mathrm{C}$ & - \\
\hline$T_{O P}^{\text {wait }}$ & Time expenditure (s) by the operator on waiting for the cobot & $\mathrm{C}$ & - \\
\hline$T_{C B}^{w a i t}$ & Time expenditure (s) by the cobot on waiting for the operator & $\mathrm{C}$ & - \\
\hline$i$ & Order line index & $\mathrm{M} \& \mathrm{C}$ & {$[1-62]$} \\
\hline$j$ & Kit container index & $\mathrm{C}$ & {$[1-4]$} \\
\hline$k$ & Compartment index & $\mathrm{C}$ & {$[1-4]$} \\
\hline$k^{\prime}$ & Index for the compartment that the cobot is working on & $\mathrm{C}$ & {$[1-4]$} \\
\hline$s$ & Shelf index & $\mathrm{M} \& \mathrm{C}$ & [1-9] \\
\hline$N_{i}$ & No. of order lines (SKUs) to kit in a work cycle & $\mathrm{M} \& \mathrm{C}$ & [27-62] \\
\hline$N_{s}$ & No. of shelf sections in picking tour & $\mathrm{M} \& \mathrm{C}$ & 9 \\
\hline$B$ & Batch size (number of kits completed in a picking tour) & $\mathrm{M} \& \mathrm{C}$ & 4 \\
\hline$C$ & Number of compartments in collaborative zone & $\mathrm{C}$ & {$[1-4]$} \\
\hline$K$ & Number of full compartments in the collaborative zone & $\mathrm{C}$ & {$[0-4]$} \\
\hline$c(k)$ & Work left (s) for the cobot in compartment $k$ & $\mathrm{C}$ & - \\
\hline$\alpha$ & Proportion of receive information and searching performed while travelling & $\mathrm{M} \& \mathrm{C}$ & 0.5 \\
\hline$\Delta$ & Order line work balance (s) between operator and cobot & $\mathrm{C}$ & - \\
\hline$\theta$ & Tool change index ( 1 if equipped tool can handle SKU $i$, otherwise 0 ) & $\mathrm{C}$ & {$[0-1]$} \\
\hline$\varepsilon$ & Failure rate (percentage) of the cobot's tool when gripping a component & $\mathrm{C}$ & {$[0.05,0.01,0.02]$} \\
\hline$d_{\text {in }}$ & One-way distance $(\mathrm{m})$ moved by cobot within collaborative zone & $\mathrm{C}$ & 0.3 \\
\hline$d_{k i t}(j)$ & Distance $(\mathrm{m})$ between the collaborative zone and kit container $j$ & $\mathrm{C}$ & {$[1.1,0.6,0.9,0.4]$} \\
\hline$L_{S}$ & Length $(\mathrm{m})$ of one shelf section & $\mathrm{M} \& \mathrm{C}$ & 1.2 \\
\hline$t_{s}$ & Time (s) for the AGV to start moving & $\mathrm{M} \& \mathrm{C}$ & 0.2 \\
\hline$t_{p}$ & Time (s) for the AGV to stop moving & $\mathrm{M} \& \mathrm{C}$ & 0.2 \\
\hline$t_{C B}^{t c}$ & Time (s) for the cobot to perform a tool change & $\mathrm{C}$ & 2 \\
\hline$t_{C B}^{g r}$ & Time (s) for the cobot to grip a component with different grippers & $\mathrm{C}$ & {$[2,1,1.2]$} \\
\hline$t_{C B}^{p l}$ & Time (s) for the cobot to place a component in a kit & $\mathrm{C}$ & 1.5 \\
\hline$q_{S K U}(i)$ & Quantity of SKU $i$ to pick & $\mathrm{M} \& \mathrm{C}$ & {$[1-8]$} \\
\hline$q_{k i t}(i, j)$ & Quantity of SKU $i$ to sort into kit $j$ & $\mathrm{C}$ & {$[1,2]$} \\
\hline$v_{O P}$ & Average travelling speed $(\mathrm{m} / \mathrm{s})$ of the operator & $\mathrm{M} \& \mathrm{C}$ & 1.1 \\
\hline$v_{C B}^{i n}$ & Linear move speed $(\mathrm{m} / \mathrm{s})$ of cobot within the collaborative zone & $\mathrm{C}$ & 1 \\
\hline$v_{C B}^{\text {out }}$ & Linear move speed $(\mathrm{m} / \mathrm{s})$ of cobot outside the collaborative zone & $\mathrm{C}$ & 2 \\
\hline
\end{tabular}

* $\mathrm{M}=$ manual kit preparation; $\mathrm{C}=$ cobot-supported kit preparation

$T_{M}=T_{O P}^{t r a v}+T_{O P}^{p i c k}+T_{O P}^{\text {sort }}$
The time spent on travelling $T_{O P}^{\text {trav }}$ in (1) can be estimated from the distance covered during the work cycle and the average travelling speed. The distance is the total length of the 
Table 2 Fitted distributions, based on experiments data, for parameters related to the operator's picking and sorting activities

\begin{tabular}{|c|c|c|c|c|}
\hline Type & Notation & Description & Model* & Fitted distribution $* *$ \\
\hline \multirow[t]{7}{*}{ Picking activities } & $t_{O P}^{I(S K U)}$ & Receive SKU information & $\mathrm{M} \& \mathrm{C}$ & 0 \\
\hline & $t_{O P}^{S(S K U)}$ & Search for SKU & $\mathrm{M} \& \mathrm{C}$ & Gamma $(0.1,6.41,0.199)$ \\
\hline & $t_{O P}^{P(S K U)_{1}}$ & Pick 1 component & $\mathrm{M} \& \mathrm{C}$ & Weibull $(0.7,1.55,0.511)$ \\
\hline & $t_{O P}^{P(S K U)_{2}}$ & Pick 2 components & $\mathrm{M} \& \mathrm{C}$ & Gamma $(0.8,3.35,0.295)$ \\
\hline & $t_{O P}^{P(S K U)_{3}}$ & Pick 3 components & $\mathrm{M} \& \mathrm{C}$ & $\operatorname{LogNormal}(1.7,1.25,1.38)$ \\
\hline & $t_{O P}^{P(S K U)_{4}}$ & Pick 4 components & $M \& C$ & Gamma $(2.4,1.0,0.867)$ \\
\hline & $t_{O P}^{C(S K U)}$ & Pick-from confirmation & $\mathrm{M} \& \mathrm{C}$ & Weibull $(0.28,1.65,0.81)$ \\
\hline \multirow[t]{7}{*}{ Sorting activities } & $t_{O P}^{I(k i t)}$ & Receive kit information & M & 0 \\
\hline & $t_{O P}^{S(k i t)}$ & Search for kits & M & Weibull $(0,2.71,0.891)$ \\
\hline & $t_{O P}^{P(k i t)_{1}}$ & Place 1 component in kit & M & Weibull $(0,2.74,0.911)$ \\
\hline & $t_{O P}^{P(k i t)_{2}}$ & Place 2 components in kits & M & Gamma $(0,5.65,0.182)$ \\
\hline & $t_{O P}^{P(k i t)_{3}}$ & Place 3 components in kits & M & LogNormal $(0,0.826,0.405)$ \\
\hline & $t_{O P}^{P(k i t)_{4}}$ & Place 4 components in kits & M & Weibull $(0,0.2,2.11)$ \\
\hline & $t_{O P}^{C(k i t)}$ & Place-to confirmation & M & Weibull $(0,4.24,1.26)$ \\
\hline Cobot-supported sorting & $t_{O P}^{P(c z)}$ & Place components in a compartment & $\mathrm{C}$ & Weibull $(0,2.74,0.911)$ \\
\hline
\end{tabular}

* $\mathrm{M}=$ manual kit preparation; $\mathrm{C}=$ cobot-supported kit preparation

**The function arguments shown in parentheses correspond to: (offset, parameter 1, parameter 2)

shelf sections $\sum L_{S}$, and each shelf section has length $L_{S}$. The operator's average travelling speed is $v_{O P}$

As has been pointed out in previous studies dealing with kit preparation [e.g. 7, 22] and order picking [24], some of the travelling time can be carried out, while other tasks are performed, for example, receiving SKU information $t_{I(S K U)}$ and searching for the SKU location $t_{S(S K U)}$. Furthermore, with the use of an AGV, the time for searching for the correct SKU in the shelves is reduced, as the AGV stops in front of the next shelf the operator should pick from and, thereby, helps the operator with the search task. The proportion of time for receiving picking information and searching for the SKU location time that can be performed while travelling is here represented by $\alpha$.

The AGV moves one shelf $s$ at a time for all shelves $N_{s}$. The operator receives picking information, $t_{I(S K U)}$, and then proceeds to search for the SKU location, $t_{S(S K U)}$, for the first SKU in each shelf. When AGV moves, there is a time required for starting, $t_{s}$, and for parking, $t_{p}$. Accordingly, the travelling time:

$T_{O P}^{\text {trav }}=N_{S} \cdot\left(t_{s}+t_{p}-\alpha \cdot\left(t_{I(S K U)}+t_{S(S K U)}\right)\right) \cdot \sum_{s=1}^{N_{s}} \frac{L_{S}}{v_{O P}}$

In accordance with Battini et al. [24], the pick task $T_{O P}^{\text {pick }}$ in (1) includes the time to receive information about the SKU (location and quantity) $t_{O P}^{I(S K U)}$, the time to search for the SKU location $t_{O P}^{S(S K U)}$, the time to pick the full quantity of the SKU
$t_{O P}^{P(S K U)_{q}}$ which differs depending on the quantity $q$, and the time to perform the pick-from confirmation of the SKU $t_{O P}^{C(S K U)}$. Similarly, as reported in Fager [23], the sorting activity $T_{O P}^{\text {sort }}$ includes the time to receive information about which kits to sort components into (location and quantity) $t_{O P}^{I(k i t)}$, the time to search for the kit $t_{O P}^{S(k i t)}$, the time to sort components by placing these in kits $t_{O P}^{P(k i t)_{q}}$ which differs depending on the quantity $q$, and the time to perform the place-to confirmation of the kit $t_{O P}^{C(k i t)}$. The time required for carrying out the picking and sorting activities can be expressed as:

$T_{O P}^{p i c k}=\sum_{i=1}^{N}\left(t_{O P}^{I(S K U)}(i)+t_{O P}^{S(S K U)}(i)+t_{O P}^{P(S K U)_{q}}(i)+t_{O P}^{C(S K U)}(i)\right)$

$T_{O P}^{\text {Sort }}=\sum_{i=1}^{N}\left(t_{O P}^{I(k i t)}(i)+t_{O P}^{S(k i t)}(i)+t_{O P}^{P(k i t)_{q}}(i)+t_{O P}^{C(k i t)}(i)\right)$

Time expenditure on manual tasks in kit preparation depends on various aspects of kit preparation design and context [4]. In this paper, to approximate time expenditure of manual tasks, statistical distributions are used. The distributions to use were identified by fitting statistical distributions to experimental data, which was collected in separate studies on manual kit preparation, as reported in Fager et al. [22] and Fager [23]. The distributions were applied to picking and sorting activities of the operator, as shown in Table 2. Time to receive SKU information $\left(t_{I(S K U)}\right)$ and time to receive kit information $\left(t_{I(k i t)}\right)$ 
Fig. 1. Overview of manual (top) and cobot-supported (bottom) kit preparation
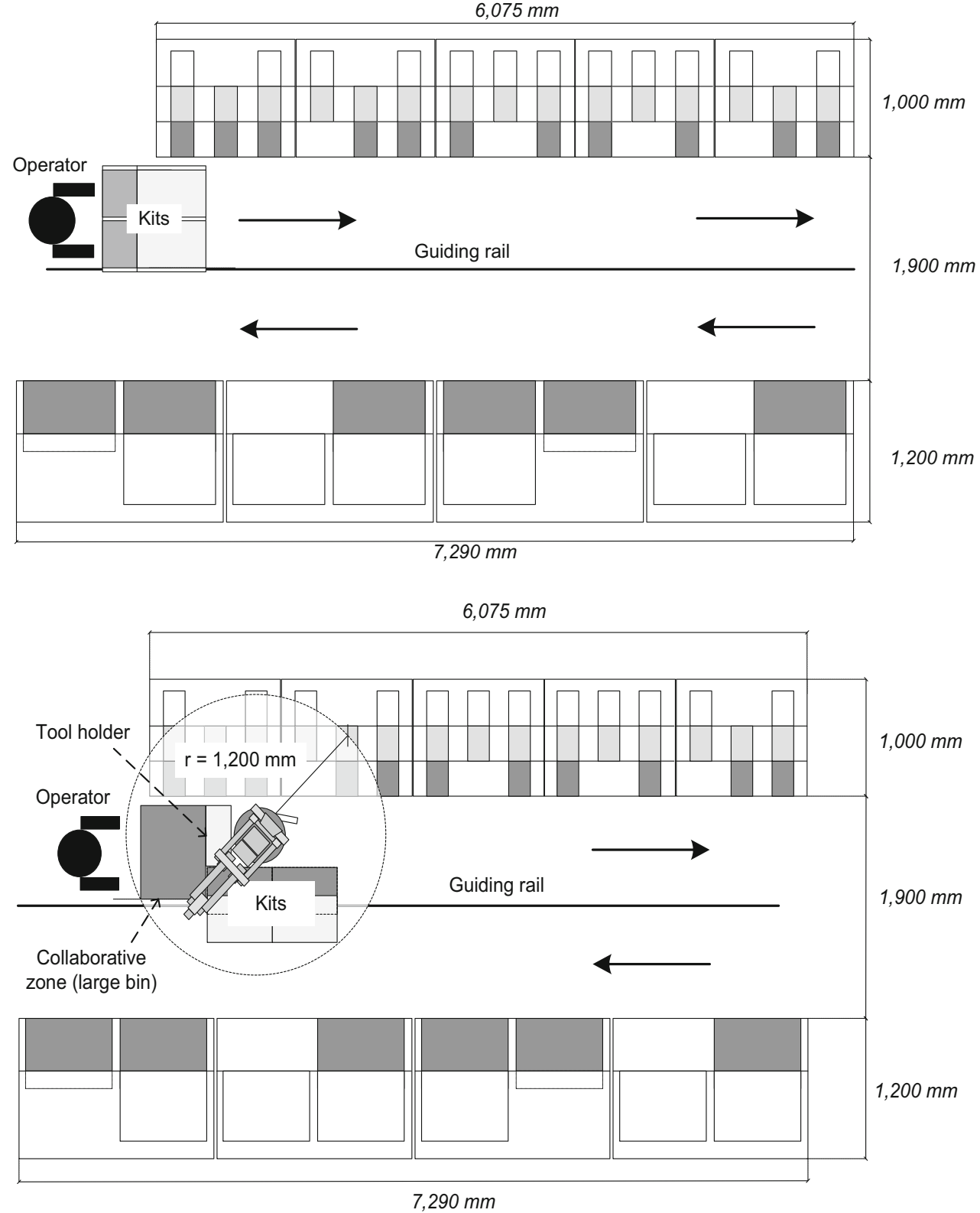

were set to 0 owing to that a pick-by-light system was considered, whereby receive information and search occur at once, in line with reasoning in Battini et al. [24].

Later, in the model for cobot-supported kit preparation, (1) is modified in order to estimate the time for the operator's activities. The cycle time of manual kit preparation can be estimated from (1) with (2), (3), and (4).

\subsection{Modelling cobot-supported kit preparation}

This section presents a mathematical model of cobotsupported kit preparation. First, a model for estimating the cycle time is presented, and thereafter models for estimating time expenditures for the operator and the cobot, respectively, are presented. The notations used are shown in Table 1 and Table 2.

\subsubsection{Cycle time of cobot-supported kit preparation}

With cobot-supported kit preparation, the operator is assisted by a cobot. For each order line $i$, the operator picks components and drops these into compartments in the collaborative zone, where after the cobot distributes the components into a batch of kits.

The cycle time, $T_{C}$, depends on the time it takes for the operator to pick components, $T_{O B}$ and the time it takes for the cobot to sort components, $T_{C B}$. While the cobot sorts order line $i, T_{C B}(i)$, the operator picks components associated with 
the next order line $i+1, T_{O P}(i+1)$. This is repeated for all $N_{i}$ order lines in the work cycle. Hence, at any given time, the operator and the cobot work on different tasks, and the operator always works ahead of the cobot.

The collaborative zone is divided into $C$ compartments so that the operator can put down all components associated with order line $i$ in a compartment. The number of compartments with components in them is $K$, and $0 \leq K \leq C$. When the operator puts components in a compartment, $K$ is increased by 1 . The cobot sets a full compartment as its current compartment $k^{\prime}$ and then retrieves components from that compartment and sorts them among the kits until the compartment is empty, whereby $K$ is decreased by 1 . The amount of work left for the cobot in compartment $k$ is $c(k)$.

If the operator is quicker with $T_{O P}(i+1)$ and finishes before the cobot has finished $T_{C B}(i)$, the operator can put components in an empty compartment and $K=K+1$ as long as $K<C$. Similarly, if the cobot finishes $T_{C B}(i)$ before the operator finishes $T_{O P}(i+1)$, the cobot can proceed with a compartment that is full since previously and $K=K-1$ as long as $K>0$. The operator has to wait $T_{O P}^{\text {wait }}(i)$ if all compartments are full $(K=C)$, and the cobot has to wait $T_{C B}^{\text {wait }}$ if all compartments are empty $(K=0)$. In this way, the cycle time $T_{C}$ is controlled by the operator as long as there are free compartments available $(K<C)$.

For the first order line, $i=1$, the cobot idles, as all $C$ compartments in the collaborative zone are empty $(K=0)$, and the cycle time is $T_{C}(1)=T_{O P}(1)$. Similarly, for the last order line, $i=N_{i}$, the operator idles, as there are no more SKUs to pick, and the cycle time is the time required for the cobot to complete the last order line $T_{C B}\left(N_{i}\right)$ and any additional work that is left in the compartments $\sum_{k=1}^{C} c(k)$. Hence, to estimate cycle time, it is necessary to keep track of the work the cobot has left in each compartment $c(1)$ to $c(C)$ throughout the work cycle. For any order line $2<i<N_{i}-1$, the cycle time $T_{C}(i)$ is controlled by the operator, $T_{O P}(i)$, as long as there are free compartments. Accordingly, the cycle time $T_{C}$ can be expressed:

$T_{C}=T_{O P}(i=1)+\sum_{i=2}^{N_{i}-1} T_{C}(i)+T_{C B}\left(i=N_{i}\right)$

The term $T_{C}(i)$ is determined by the work balancing between the operator and the cobot during the work cycle. If the operator finishes first and there are no free compartments to drop off components in $(K=C)$, the operator has to wait until the cobot has finished its current task $c\left(k^{\prime}\right)$, whereby the cobot controls the cycle time. Similarly, if the cobot finishes first and there are no more compartments with components in them $(K=0)$, then the cobot must wait until the operator has finished the task. Hence, the compartments allow that the operator can continue even if the cobot has not yet finished its work.
Whenever the cobot falls behind, the cobot builds a back$\log$ of work that it can work on reducing when there are order lines where the cobot finishes before the operator. This dynamic between the cobot and the operator depends on whether the operator or the cobot finishes their task first, here represented by $\Delta$.

For the first order line in $(1), T_{O P}(i=1)$, the addition to the cycle time can be estimated in accordance with Table 3 .

For order lines between 1 and $N_{i}$, corresponding to the second term in (5), the cycle time $T_{C}(i)$ depends on the time difference for the operator and the cobot to complete their current tasks, represented by $\Delta=T_{O P}(i)-c\left(k^{\prime}\right)$. When $\Delta<0$, the operator is quicker than the cobot, and when $\Delta>0$, the cobot is quicker than the operator. This can be implemented in line with Table 3.

When $\Delta<0, T_{C}(i)$ depends on whether there are free compartments available $(K<C)$ or not $(K=C)$. The logic can be described as follows. If there are free compartments in the collaborative zone $(K<C)$, the operator time is added to the cycle time, $T_{C}(i)=T_{O P}(i)$, as the components can be put away in an empty compartment regardless of whether the cobot has finished or not. The operator finishing first does, however, lead to one more compartment becoming full $(K=K+1)$ since the operator puts the components of order line $i+1$ in an empty compartment, adding $T_{C B}(i)$ to one of the $C$ compartments. Here, $K=$ $K+1$ as long as $K<C$. However, when $K=C, T_{C}(i)$ is equal to the time required by the cobot for its current compartment $c\left(k^{\prime}\right)$, as the cobot needs to free up a compartment before the operator can continue, and the operator has to wait $\left(T_{O P}^{\text {wait }}=a b s(\Delta)\right)$. This logic is expressed as shown in Table 3.

When $\Delta<0$, i.e. the cobot finishes its work before the operator, the cobot has time to spare and can catch up on backlogged work in the $k$ compartments, to free up compartments. The cobot's backlog is iteratively calculated at each new order line $i$ for each of the $C$ compartments. However, the iteration stops once the operator has caught up with the cobot. The logic shown in Table 3 can be used to keep track of how many compartments are freed up.

This frees up compartments between the cobot and the operator. If the cobot has no backlog, i.e. $K=0$, the cobot idles $\left(T_{C B}^{\text {wait }}\right)$ until the operator finishes order line $i+1$. A restriction here is that the order lines must be completed in the same order the operator carries out the order lines for the components to end up in the correct order within the kit containers. However, the operator may put the components in any free compartment within the collaborative zone but should prioritise compartments for which the distance is shorter.

For the last order line $N_{i}$, corresponding to the third term in (5), the cycle time is calculated from the work the cobot has 
Table 3 Example logic for model implementation

\begin{tabular}{|c|c|}
\hline \multicolumn{2}{|l|}{ Cycle time at order line $i=1$} \\
\hline If $i=1$ Then & Check that order line index is 1 \\
\hline$c(1)=T_{C B}(i)$ & Compartment 1 gets $\mathrm{CB}(1)$ work added to it \\
\hline $\begin{array}{l}\text { Compartment } 1 \text { gets } \mathrm{CB}(1) \text { work added to it } \\
\text { OP puts components in a compartment }\end{array}$ & OP puts components in a compartment \\
\hline $\begin{array}{l}\text { OP puts components in a compartment } \\
\text { Set the full compartment as the cobot's current compartment }\end{array}$ & Set the full compartment as the cobot's current compartment \\
\hline \multicolumn{2}{|r|}{ Cycle time controlled by OP } \\
\hline$T_{C B}^{\text {wait }}(i)=T_{O P}(i)$ & The cobot waits while OP carries out the first order line \\
\hline \multicolumn{2}{|l|}{ End If } \\
\hline Cycle time at order line $1<i<N_{i}$ & \\
\hline Select Case $\Delta$ & \\
\hline Case $\Delta<0$ & Check if $\Delta$ is negative, i.e. is OP faster than CB? \\
\hline If $K<C$ Then & Check that there is a free compartment available \\
\hline For $k=1$ To $C$ & Find a free compartment for OP to put components in \\
\hline If $c(k)=0$ Then & Check if compartment $k$ is empty... \\
\hline$c(k)=T_{C B}(i)$ & OP puts the components in compartment $k$ \\
\hline$K=K+1$ & Register a full compartment \\
\hline Exit For & \\
\hline End If & \\
\hline Next $k$ & \\
\hline$c\left(k^{\prime}\right)=c\left(k^{\prime}\right)-T_{O P}(i)$ & Calculate the work CB has left in its current compartment \\
\hline$T_{C}=T_{C}+T_{O P}(i)$ & Cycle time is expanded by OP \\
\hline ElseIf $K=C$ Then & Check if all compartments are full \\
\hline$T_{C}=T_{C}+c\left(k^{\prime}\right)$ & OP waits until CB finishes its current compartment \\
\hline$T_{O P}^{\text {wait }}=T_{O P}^{\text {wait }}+a b s(\Delta)$ & Wait time for OP \\
\hline$c\left(k^{\prime}\right)=T_{C B}(i)$ & OP puts components into the just emptied compartment \\
\hline For $k=1$ To C & Find CB's next task \\
\hline If $c(k)>0$ Then & Check if compartment $\mathrm{j}$ is non-empty \\
\hline$k^{\prime}=k$ & Set compartment $k$ as CB's current compartment $k^{\prime}$ \\
\hline Exit For & \\
\hline End If & \\
\hline Next $k$ & \\
\hline End If & \\
\hline Cycle time at order line $i=N_{i}$ & \\
\hline If $i=N_{i}$ Then & \\
\hline For $k=1$ to $C$ & \\
\hline$T_{C}=T_{C}+c(k)$ & \\
\hline$c(k)=0$ & \\
\hline Next $k$ & \\
\hline End if & \\
\hline Determine $\Delta$ at order line $i$ & \\
\hline If $1<i<N_{i}$ Then & Check that order line index is between 1 and $N_{i}$ \\
\hline$\Delta(i)=T_{O P}(i)-c\left(k^{\prime}\right)$ & Difference between OP(i) and the cobot's current task $c\left(k^{\prime}\right)$. \\
\hline Determine $K$ at order line $i$ & \\
\hline Case $\Delta(i) \geq 0$ & Check if $\Delta$ is positive, i.e. is OP slower than $\mathrm{CB}$ ? \\
\hline$T_{C}=T_{C}+T_{O P}(i)$ & \\
\hline If $c\left(k^{\prime}\right)>0$ Then & Check if $\mathrm{CB}$ has work left on its current task \\
\hline$c\left(k^{\prime}\right)=0$ & Set the current task to zero, since it is now complete \\
\hline If $K>0$ Then & \\
\hline $\mid k=k-1$ & Register that a compartment was freed up \\
\hline Else & \\
\hline $\mid k=0$ & \\
\hline End If & \\
\hline If $K>0$ Then & Check if there are full compartments \\
\hline For $k=1$ To $C$ & \\
\hline If $c(k)>0$ Then & \\
\hline$k^{\prime}=k$ & Set the next full compartment as CB's current compartment $k^{\prime}$ \\
\hline If $c\left(k^{\prime}\right)<\Delta(i)$ Then & Check if the work left with compartment $k^{\prime}$ is less than $\Delta$ \\
\hline$\Delta(i)=\Delta(i)-c\left(k^{\prime}\right)$ & Reduce $\Delta$ by the work left in compartment $k^{\prime}$ \\
\hline$c\left(k^{\prime}\right)=0$ & \\
\hline If $K>0$ Then & \\
\hline$K=K-1$ & Register that a compartment was freed up \\
\hline ElseIf $K=0$ then & \\
\hline $\mid T_{C B}^{\text {wait }}=\Delta(i)$ & Add waiting time to $\mathrm{CB}$ \\
\hline End If & \\
\hline ElseIf $c\left(k^{\prime}\right)>\Delta(i)$ Then & Check if the work left with compartment $k$ is greater than $\Delta$ \\
\hline$c\left(k^{\prime}\right)=c\left(k^{\prime}\right)-\Delta(i)$ & Reduce the work left in compartment $k^{\prime}$ by $\Delta$ \\
\hline$\Delta(i)=0$ & \\
\hline Exit For & \\
\hline End If & \\
\hline End if & \\
\hline | Next $k$ & \\
\hline ElseIf $K=0$ Then & Check if all compartments are empty \\
\hline $\mid T_{C B}^{\text {wait }}=\Delta(i)$ & Add waiting time to $\mathrm{CB}$ \\
\hline End If & \\
\hline End If & \\
\hline For $k=1 \mathrm{To} C$ & \\
\hline If $c(k)=0$ Then & Check if compartment $k$ is empty \\
\hline$c(k)=T_{C B}(i)$ & OP puts components in compartment $k$ \\
\hline$K=K+1$ & Register that a compartment was filled \\
\hline Exit For & \\
\hline End If & \\
\hline Next $k$ & \\
\hline
\end{tabular}


left to do in the compartments, in addition to the last order line. The logic is expressed as shown in Table 3.

\subsubsection{Model of cobot activities}

Robot-supported picking has been modelled before [see, e.g. 7], but the sort task related to kit preparation has not been considered. Here, a combination of the reasoning presented by Boudella et al. [7] and observations from a laboratory setup are applied to develop a model for cobot-supported sorting.

The time requirement of the cobot's activities $T_{C B}$ is composed of the travelling time $T_{C B}^{\text {trav }}$, time required by the visionsystem $T_{C B}^{v i s}$, time for performing tool change $T_{C B}^{t o o l}$, time for gripping components in compartments $T_{C B}^{\text {grip }}$, and time for sorting components into kits $T_{C B}^{\text {sort }}$.

$T_{C B}=T_{C B}^{\text {trav }}+T_{C B}^{v i s}+T_{C B}^{\text {tool }}+T_{C B}^{g r i p}+T_{C B}^{\text {sort }}$

Here, the travel time $T_{C B}^{\text {trav }}$ corresponds to the travel time of the operator, but as the cobot can work while travelling, the travelling time does not add to the cobot's time requirement, and $T_{C B}^{t r a v}$ is always zero. Furthermore, since the vision system is mounted above the collaborative zone, image processing can happen continually, and the $T_{C B}^{v i s}$ will too always be zero.

With tool changing time $T_{\text {tool }}^{C B}$, the same amount of time for all tool changes, $t_{C B}^{t c}$, but whether a tool change have to be made depends on whether two consecutive SKUs require the same tool or not. This is evaluated in $\theta$, which is 1 when no tool change is required and 0 if a tool change is required, for order line $i$. Moreover, the cobot always starts with the correct tool for the first order line $\theta(1)=1$. Accordingly:

$$
T_{C B}^{t o o l}=t_{C B}^{t c} \cdot \sum_{i=2}^{N_{i}}(1-\theta(i))
$$

When retrieving components from the compartments in the collaborative zone $T_{C B}^{g r i p}$, the failure rate of picking the component on the first try, $\varepsilon$, depends on the type of gripper and the component characteristics [7]. The gripping time $t_{C B}^{g r}$ is the same for any component gripped successfully on the first try, with the same tool. For each order line $i$, the cobot has $q_{S K U}(i)$ components to retrieve from the collaborative zone. Hence, the gripping time in the collaborative zone:

$$
T_{C B}^{g r i p}=t_{c b}^{g r} \cdot \sum_{i=1}^{N_{i}}\left(q_{S K U}(i) \cdot(1+\varepsilon(i))\right)
$$

The time required by the cobot for the sorting activity, $T_{C B}^{s o r t}$, depends both on from which compartment components are picked up and on which kit container the components are placed in. Furthermore, the cobot has to move at reduced speed inside the collaborative zone, $v_{C B}^{\text {in }}$, for safety, as opposed to when outside of the collaborative zone, $v_{C B}^{\text {out }}$. Here, it is assumed that after the cobots grips a component from the collaborative zone, the cobot moves the closest route to get outside the collaborative zone, $d_{i n}$, and vice versa when entering the collaborative zone. Once outside the collaborative zone, the cobot moves in a straight line of length $d_{k i t}(j)$ to the centre of kit container $j$. The cobot's movement during the sorting activity is shown in Fig. 2. Each kit $j$ of all kits in the batch $B$ should receive $q_{k i t}(i, j)$ components of SKU $i$. The time for placing a component into a kit container $t_{C B}^{p l}$ is the same for all components and gripper types. Moreover, the cobot always returns the same way to the collaborative zone after sorting a component. Accordingly:

$T_{C B}^{\text {sort }}=\sum_{i=1}^{N_{i}} \sum_{j=1}^{B} q_{k i t}(i, j)\left(\frac{2 \cdot d_{i n}}{v_{C B}^{\text {in }}}+\frac{2 \cdot d_{k i t}(j)}{v_{C B}^{\text {out }}}+t_{C B}^{p l}\right)$

The cobot's time requirement from (6) can now be estimated with (7), (8), and (9).

\subsubsection{Model of the operator's activities}

The operator's time expenditure in cobot-supported kit preparation is similar to the manual setup. The travelling time, $T_{O P}^{t r a v}$, is estimated in accordance with (2) and the picking time $T_{O P}^{p i c k}$ in accordance with (3). The sorting activity is now simpler, and instead of having to sort components between kit containers and performing place-to confirmations, the operator puts all components in a compartment in the collaborative

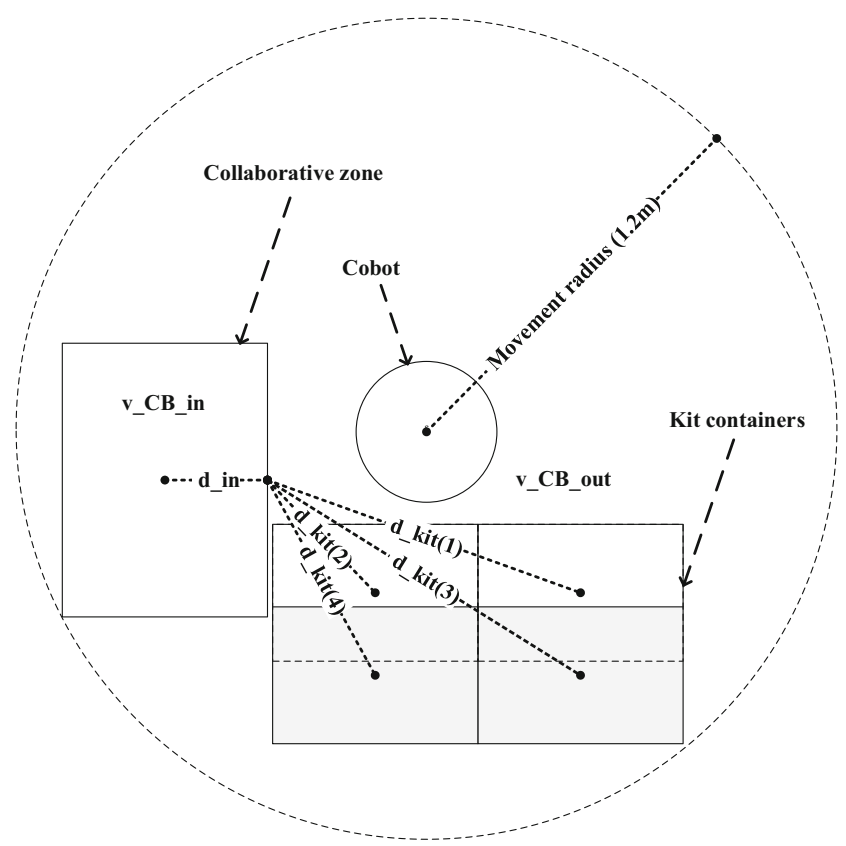

Fig. 2 Overview of the cobot's movement during the sorting activity 
zone, represented by $t_{O P}^{P(c z)}$. In line with (4), placing components in a compartment in the collaborative zone can be approximated with a Weibull distribution, as shown in Table 2.

\section{Model application}

As previously stated, the purpose of this paper is to identify the potential of a cobot to support time-efficient batch preparation of kits. Therefore, in this section, the mathematical model is applied in a case example to indicate differences with respect to cycle time and time allocation among activities of cobot-supported kit preparation when compared with kit preparation performed manually. The case example is based on a laboratory setup of kit preparation that was used in experiments (see Fager et al. [22] and Fager [23]). The setup was constructed to mimic industrial kit preparation in the automotive industry.

Each result presented in this section is based on preparation of 500 kits, which roughly corresponds to the production volume of 2 work shifts. The kits were prepared in batches of four kits, and each kit's content was based on the distributions of high, medium, and low runners within the component families (see Sect. 4.1).

\subsection{Description of case example}

An overview of the layout of the kit preparation process considered here is shown in Fig. 1, and the values used in the numerical example are shown in Table 1 and Table 2. The values for the operator's activities were estimated based on earlier experiments dealing with manual picking from Fager et al. [22], Fager [23], and Battini et al. [24]. The parameters for cobot picking activities were estimated based on Boudella et al. [7], observations in a laboratory, and on discussions with a cobot developer.

The kit preparation workspace is organised as an openended picking aisle. The process involves 51 different component variants, representing 15 component families in the product structure (2-6 component variants per component family). The components are typical for automotive assembly and have varying characteristics, ranging from cords and hoses to bearings and attachments. Each component family is composed of one or two high runner variants (50 to $80 \%$ of the volume) and medium or low runner variants (10\% of the volume). The 51 component variants are stored in boxes of size $200 \times 300 \times 200 \mathrm{~mm}$ in three-level flow racks on each side of the picking aisle. A complete kit contains at least 1 component from each of the 15 component families.

In the process, four kit containers (boxes of size $300 \times 400 \times 200 \mathrm{~mm}$ ) are transported on an $\mathrm{AGV}$, moving in front of an operator throughout the work cycle, stopping at each shelf section where components should be collected. The work cycle always starts at the shelf section with the smaller boxes, moving in a U-pattern complete the work cycle. Hence, the operator always retrieves components from the shelves to the left side of the AGV. The picker is guided by a pick by light system, and light indicators with associated displays are mounted above each storage location and above each kit container on the AGV. For each order line, corresponding to one SKU, the light indicator of the corresponding SKU lights up, and the operator retrieves the number of components of the SKU that is indicated on the display next to the light indicator. Once all components have been extracted, the operator presses a button next to the light indicator and shuts it off. These activities are performed the same regardless if kit preparation is performed manually or cobot-supported.

With manual kit preparation, light indicators at the kit containers that should receive components light up once the operator has performed SKU confirmation. The operator sorts the components into one kit at a time and presses the button above the kit container to shut of the light indicator after all components have been place in a kit. This is repeated until all components have been sorted into kits. See top half of Fig. 1.

With cobot-supported kit preparation, once components have been picked, the operator puts the components in the collaborative zone on the AGV, from which the cobot retrieves the components and sorts them into the kits; see bottom half of Fig. 1. The collaborative zone is made up of a large bin $(600 \times 800 \times 200 \mathrm{~mm})$ that can be divided in up to four compartments. The operator can place all components of an SKU in any empty compartment. A compartment can only contain a single part number at once. The cobot retrieves components from the collaborative zone, one compartment and component at a time, and distributes them among the kit containers in accordance with the production plan. The cobot has three different grippers at its disposal (servo-electric 2-jaw, pneumatic, and magnetic) to be able to grip different components.

\subsection{Time comparison of manual and cobot-supported kit preparation}

In Fig. 3, a comparison of manual and cobot-supported kit preparation is shown. The comparison was made based on the example values in Table 1 and considers cobot-supported kit preparation when a single compartment is used in the collaborative zone. The comparison shows that the two setups display similar time requirements for kitting components, while the cobot-supported setup shows slightly less variability owing to the more consistent pacing of the cobot compared to when all activities are performed manually.

Looking into how the operator and the cobot allocate time during the work cycle in the two setups, Fig. 4 shows how time is allocated among various activities. With manual kit preparation (left column in Fig. 4), the operator spends most time on picking 
Fig. 3 Average time per kitted component for manual kit preparation $\left(\mathbf{T}_{\mathbf{M}}\right)$ and cobotsupported kit preparation $\left(\mathbf{T}_{\mathbf{C}}\right)$ using one compartment in the collaborative zone. The boxes represent one standard deviation from the average (centre line in each box). The flat-end lines above and below each box indicate maximum and minimum

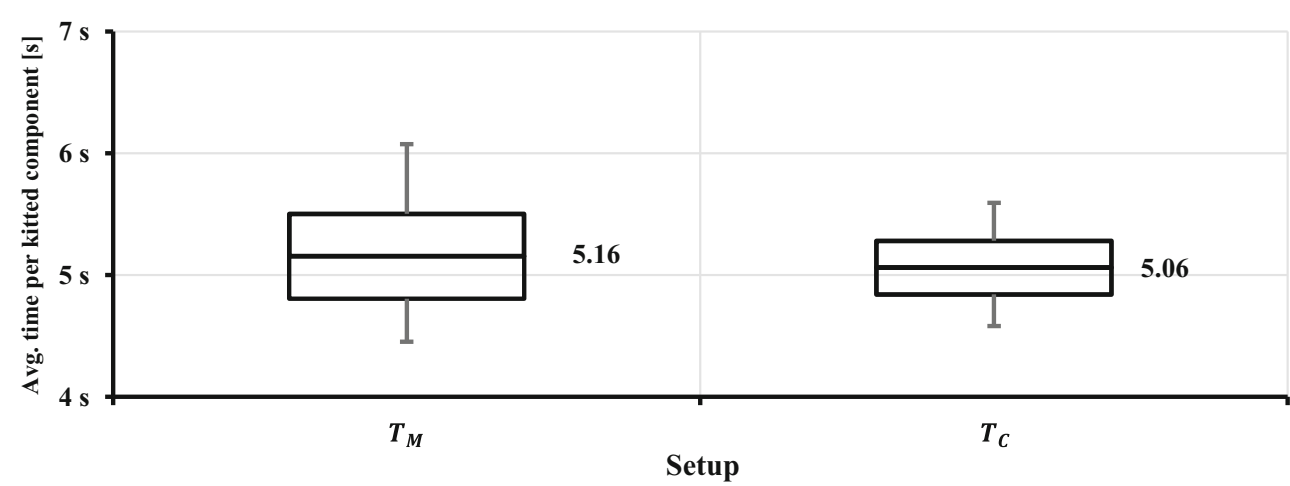

components (29\%). Kit confirmation time is considerable (19\%), as is search time (14\%) and placing components in kits $(13 \%)$. Only about $7 \%$ of the time is spent on travelling, as the preparation area is dense, and the operator is supported by the AGV which stops in front of each shelf with SKUs to be picked. SKU confirmation time is $10 \%$ of the total time, and $8 \%$ of the time is spent on searching for the correct kit container.

The middle column in Fig. 4 shows how time is allocated among activities for the operator in cobot-supported kit preparation. Here, the operator has fewer activities to carry out than if the process is performed manually, and more of the operator's time is spent on picking components (34\%). The time spent by the operator on placing components in compartments the collaborative zone is $11 \%$, and the time spent on travelling is small $(8 \%)$, due to the high density of the picking area. Furthermore, SKU confirmation time now accounts for $12 \%$ of the operator's time expenditure, and $16 \%$ is search time. Furthermore, the operator waits for the cobot for $19 \%$ of the time, which can be useful when, for example, handling empty storage packaging or performing checks of newly replenished materials.

As the right column in Fig. 4 shows, the cobot spends most of its time $(48 \%)$ on the sort task, by placing components one at a time in the kits. The tool change time makes up $12 \%$, and the proportion of time spent on the collaborative task - retrieving components from and moving within - the collaborative zone is $39 \%$. The wait time of $1 \%$ occurs during the first order line when there are no components in the collaborative zone.

Figure 4 shows that when the cobot supports the operator, the operator can focus more on picking components from the shelves and less on sorting components into kits. With the operator, the time spent on activities related to picking, searching for SKUs, and performing SKU confirmations is related to kit commonality, as the more diverse the range of SKUs to kit are, the more times these activities must be performed. Kit commonality also affects the cobot: as more diverse kits means that there are fewer components on average in each compartment to sort into kits, and since the cobot only can handle one component at a time, this should allow the cobot to better keep up with the operator's pace. Conversely, when kit commonality is high, such as when standard kits are produced, there is on average more components in each compartment for the cobot to handle, and its efficiency relative to the operator should be less. This would also be the case when more components of each SKU are kitted. These aspects, i.e. kit commonality and components per SKU, are considered later in the analysis with respect to a comparison between manual and cobot-supported kit preparation.

The ability for the operator and the cobot to continuously work depends on that compartments in the collaborative zone are available for the operator to put components in and for the cobot to collect components from. Hence, all activities in Fig.
Fig. 4 Time allocation among activities in manual kit preparation $(\mathrm{M})$ for the operator (left column) and in cobotsupported kit preparation (C) for the operator (middle column) and for the cobot (right column)
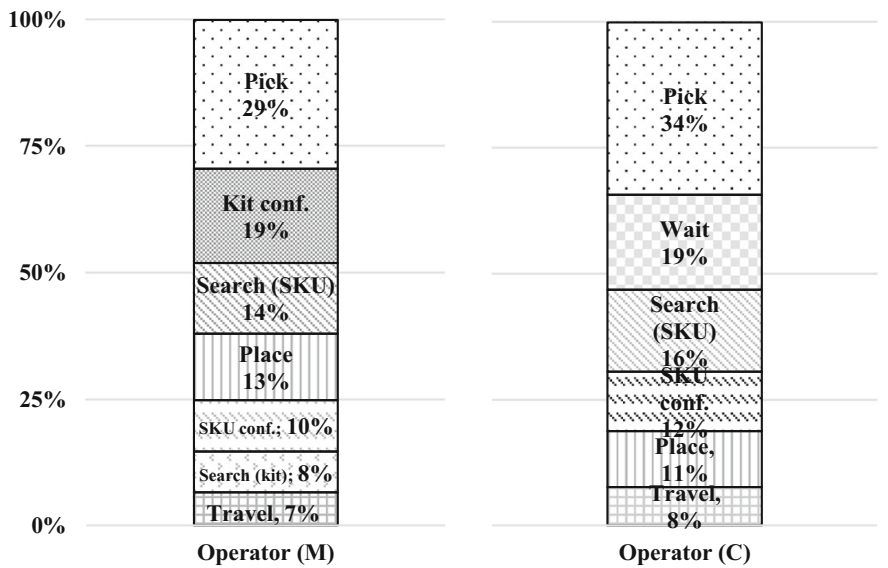
4 which are related to handling of components, e.g. picking and sorting, are affected by the number of compartments that are available in the collaborative zone. Introducing more compartments in, or removing compartments from, the collaborative zone can hence affect time efficiency associated with the process and thereby makes up a relevant consideration. This is considered in the next section.

\subsection{Analysing the effect of the number of compartments in the collaborative zone}

An important aspect of keeping the work cycles of the operator and the cobot synchronised is that neither party have to wait for the other. If the operator has only one compartment to put components in, the operator has to wait every time when the cobot has not been able to sort the components from the previous order line. However, if more compartments are introduced in the collaborative zone, then the operator can make use of a free compartment any time the cobot has not been able to sort all components. Similarly, if the cobot finishes its job quickly, it can work on reducing the backlog in other compartments instead of waiting for the operator to retrieve more components. Figure 5 shows the cycle time when one to four compartments are used in the collaborative zone.

As Fig. 5 shows, using more compartments in the collaborative zone improves the average cycle time expressed as the average time per kitted component. It seems that benefit of using more compartments lessens when more than two compartments are used in the collaborative zone, but that efficiency improves slightly even when four compartments are used instead of three. Furthermore, using four compartments seems to result in a more stable outcome. Therefore, four compartments are considered in the last part of the analysis where manual kit preparation is compared with cobot-supported kit preparation with respect to kit commonality and components per SKU.

\subsection{Cobot-supported kit preparation under varying operating conditions}

There are many contextual factors that can affect time expenditures in kit preparation, and any company makes up a unique set of contextual characteristics. Two central aspects with respect to kit preparation and robot-supported picking is kit commonality - meaning how similar the contents are between kits in a kit preparation process [2] - and the number of components picked per SKU [7]. Figure 6 shows the cycle time of manual and cobot-supported kit preparation under varying analysis settings with respect to kit commonality and quantity to kit per SKU, in accordance with Table 4.

From Fig. 6, it can be seen that the average time per kitted component is lower for cobot-supported kit preparation under analysis setting 1 and that manual kit preparation is comparatively more efficient under analysis settings 2,3 , and 4 . For all the analysis settings, cobot-supported kit preparation has, however, a less variable outcome. For the work balancing between the operator and cobot, analysis settings 2, 3, and 4 means that the operator can pick several components at oncewhich improves efficiency with respect to the operator-while the cobot gets a variable amount of components served in each compartment, causing it to occasionally catch up and occasionally halt the process, and the four compartments act as a buffer. With identical kit contents and two components kitted per SKU, there is likely too many components in each compartment for the cobot to handle, and the process is halted a while into the picking tour where the operator must wait for the cobot.

\section{Discussion and managerial implications}

The paper made use of a modelling approach to analyse and compare manual and cobot-supported kit preparation. The
Fig. 5 Average time per kitted component of cobot-supported kit preparation, when using different numbers of compartments in the collaborative zone. The boxes indicate one standard deviation from the average (centre line), and the flat-end lines indicate maximum and minimum

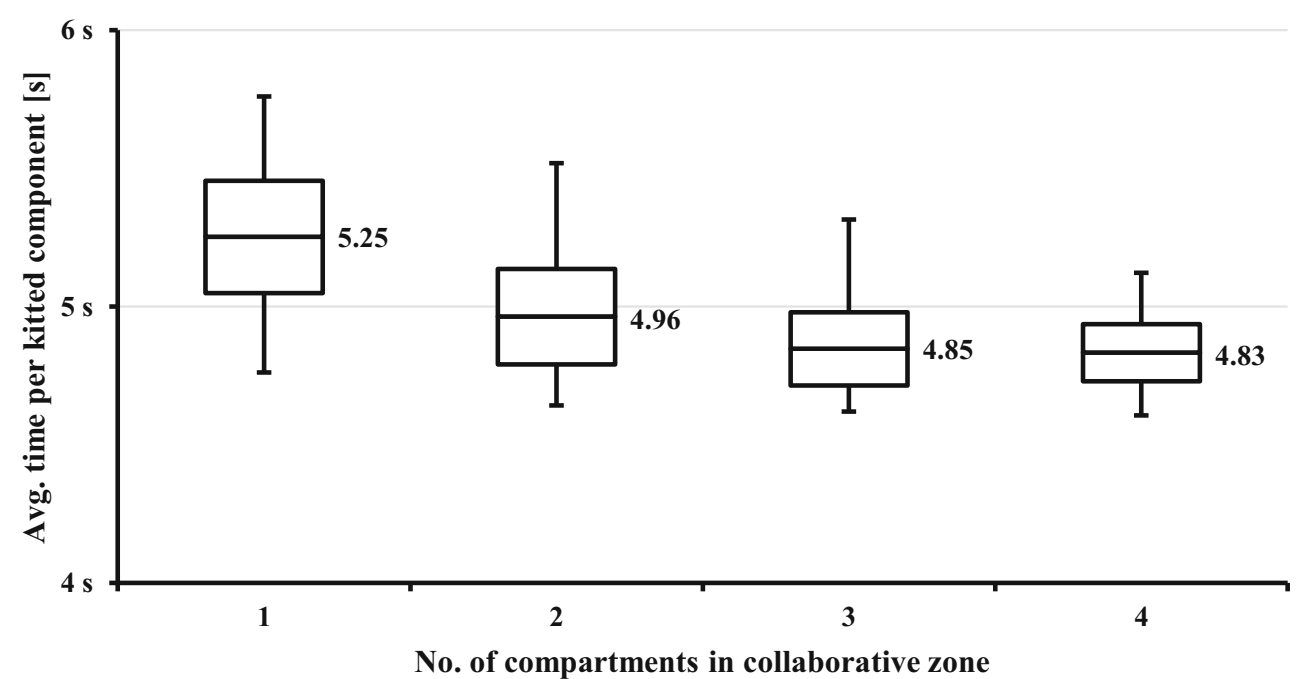


Fig. 6 Average time per kitted component of manual (M) and cobot-supported (C) kit preparation under varying operating conditions with respect to kit commonality and quantity to kit per SKU, in accordance with Table 3. The boxes indicate one standard deviation from the average (centre line), and the flatend lines indicate maximum and minimum

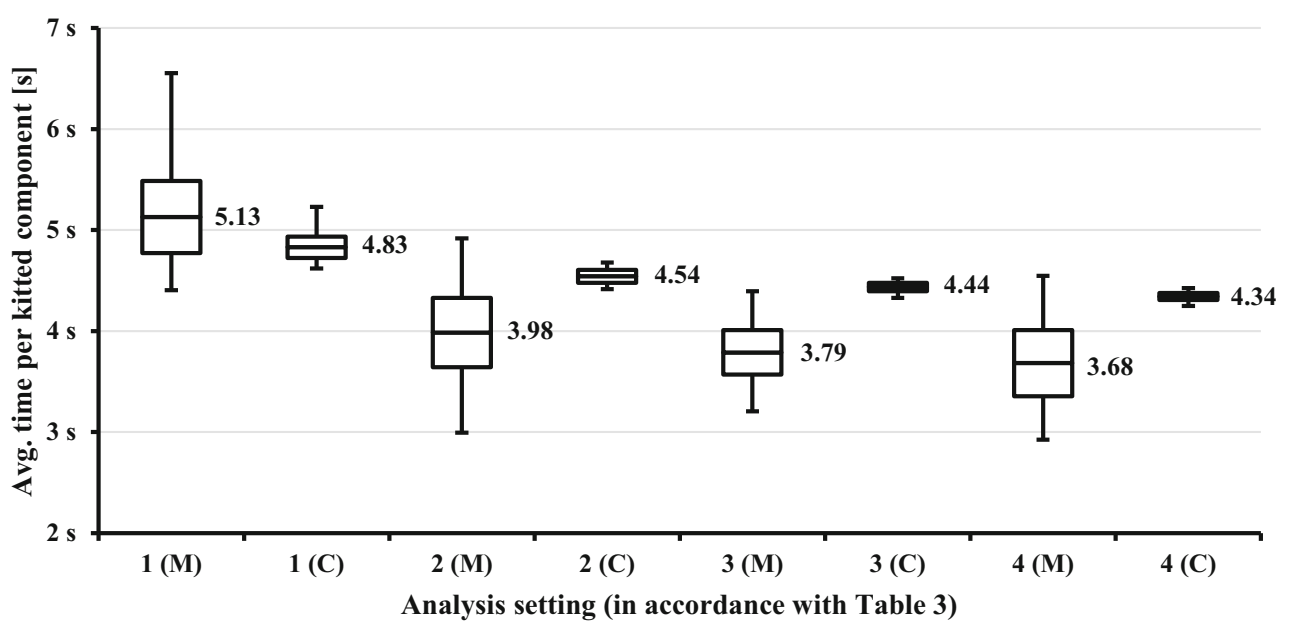

results from the paper align well with previous studies that have considered cobots applied to kit preparation [e.g. 7, 8]. An important contribution made by this paper is the model for how a cobot can support kit preparation by sorting components into a batch of kits. This has not been available in previously, as the focus has been on picking components from shelves or on robots that perform work separate from operators. The application of a cobot to support the sorting task with batch preparation of kits is beneficial for kit quality, as it removes risks of human errors when sorting components among kits.

The number of compartments used in the collaborative zone with cobot-supported kit preparation showed to have a substantial effect. Here, more compartments allowed the operator to continue retrieving SKUs from the shelves even if the cobot could not keep up. The cobot fell behind the operator when there were many components of an SKU picked at once, as the cobot can only handle a component at a time, while the operator can pick several components at once. In this way, the operator benefits more from approaches whereby more components are picked simultaneously, such as when kits have high commonality or when kits involve multiple components of the same SKU. However, cobot support seems to provide a more stable outcome even under settings where manual kit preparation is preferable from an efficiency standpoint.

In the numerical example of the model, the operator's activities were modelled by means of fitting distributions to experimental data. This approach brings variability of time expenditures similar to what occurs in live kit preparation in industry. Furthermore, variability of time expenditures for various activities is important to account for with respect to the cobot application considered in the paper, as the work balancing between the operator and the cobot governs the efficiency outcome. The use of fitted distributions should be replicable when the application is considered in various industrial contexts, and the benefit of this approach is that data over manual activities can be retrieved from the settings where at the cobot application is considered for use, allowing for an assessment of the performance to expect.

With the cobot application considered in the paper, some parameters stood out as important with respect to the outcome of the kit preparation. Some of these relate to the distances and speeds at which the cobot moves, both within and outside the collaborative zone. These parameters vary between cobot types but can also vary between application contexts, where some contexts allow shorter distances and higher speeds than others. It is important to consider such aspects before implementation, not the least from a perspective of safety and current technological standards. The model presented in the paper makes up an appropriate tool for such evaluations.

While the paper has focused on the context of kit preparation, which normally is performed in dense picking areas and involves picking at frequency with small travelling distances [4], it is interesting to consider how the cobot application studied in the paper applies in other contexts. One interesting context is in warehouses and order picking. Here, distances in between SKUs would likely be longer, and the cobot should
Table 4 The settings used in the analysis in Fig. 5

\begin{tabular}{lll}
\hline Analysis setting & Kit commonality & $\begin{array}{l}\text { No. of components } \\
\text { kitted per SKU }\end{array}$ \\
\hline 1 & Different kit contents & 1 component per SKU \\
2 & Identical kit contents & 1 component per SKU \\
3 & Different kit contents & 2 components per SKU \\
4 & Identical kit contents & 2 components per SKU \\
\hline
\end{tabular}


have more time available for its work when the operator is travelling and thereby have less trouble keeping up with the operator. Other settings whereat the application could prove fruitful to consider can be in association with spare parts kitting and order picking, repair and maintenance, or business to business industries that involves diverse orders of products and components.

A limitation of the approach is that the model and the experiment where from the experimental data were retrieved are simplifications of a real industrial setting and there may be other aspects not covered by the model that needs consideration with respect to implementation of the cobot application. For example, there may be unsuitable component characteristics for cobot picking [see e.g. 7] or different safety regulations compared with a laboratory environment, since other people than the operator may be present at the preparation area. However, the model presented in the paper, as well as the experiments from which the data used for the example values were retrieved, have been developed in close association with industrial parties that all make use of kit preparation. Thereby, the simplifications on which the model and the data are based have been made with one foot in theory and one foot in practice. Still, a comprehensive analysis must be carried out before implementation is considered.

\section{Conclusions}

This paper has modelled and compared kit preparation when the work is performed manually with an application whereby kit preparation is performed collaboratively by an operator and a cobot. The paper advances the analysis of an application of cobots in kit preparation that was presented in Fager et al. [10] by considering how the number of compartments in the collaborative zone, kit commonality, and number of components per SKU affect the comparison. The paper acknowledged that cobots applied to kit preparation has not received much previous attention in literature, apart from a few recent studies by Boudella et al. [7] and Coelho et al. [8]. The available literature presented relevant input for the paper, and the paper's results align well with these previous studies. This paper extends previous knowledge by showing how cobots can be applied in kit preparation to support the operator with the sorting task with batch preparation of kits. Furthermore, the paper considered how an operator and a cobot interact during the work cycle with respect to kit preparation time efficiency, which is new in literature.

The comparison between manual and cobot-supported kit preparation presented in the paper was made with respect to the time efficiency of kit preparation, which is central for kit preparation in industrial settings. The findings suggest that the use of cobots to support the sort task in kit preparation offers a higher time efficiency for low to moderate amounts of components to be sorted per SKU and that it results in a more stable outcome than kit preparation performed manually. This is because the operator is relieved of activities associated with sorting components into kits. Furthermore, cobotsupported sorting can remove the risk of human errors with respect to misplacing components between kits, which is important when kitting is used in industry [5, 6].

Managers may use the model to assess whether a cobotsupported kit preparation may be feasible in their own systems. Future research should explore how cobot-supported application performs in other settings, for example, warehouse order picking, and consider how, for example, longer travelling distances affect the application's performance. Aside from efficiency, future research should also consider how collaborative applications affect other performance areas of kit preparation, for example, flexibility, ergonomics, and cost.

Acknowledgements Open access funding provided by Chalmers University of Technology.

Open Access This article is licensed under a Creative Commons Attribution 4.0 International License, which permits use, sharing, adaptation, distribution and reproduction in any medium or format, as long as you give appropriate credit to the original author(s) and the source, provide a link to the Creative Commons licence, and indicate if changes were made. The images or other third party material in this article are included in the article's Creative Commons licence, unless indicated otherwise in a credit line to the material. If material is not included in the article's Creative Commons licence and your intended use is not permitted by statutory regulation or exceeds the permitted use, you will need to obtain permission directly from the copyright holder. To view a copy of this licence, visit http://creativecommons.org/licenses/by/4.0/.

\section{References}

1. Bozer YA, McGinnis LF (1992) Kitting versus line stocking: a conceptual framework and a descriptive model. Int J Prod Econ 28(1):1-19

2. Caputo AC, Pelagagge PM, Salini P (2018) Economic comparison of manual and automation-assisted kitting systems. IFACPapersOnLine 51(11):1482-1487

3. Medbo L (2003) Assembly work execution and materials kit functionality in parallel flow assembly systems. Int J Ind Ergon 31(4): 263-281

4. Hanson R, Medbo L (2019) Man-hour efficiency of manual kit preparation in the materials supply to mass-customised assembly. Int J Prod Res 50(4):1115-1125

5. Caputo AC, Pelagagge PM, Salini P (2017) Modeling errors in parts supply processes for assembly lines feeding. Ind Manag Data Syst 117(6):1263-1294

6. Caputo AC, Pelagagge PM, Salini P (2017) Modelling human errors and quality issues in kitting processes for assembly lines feeding. Comput Ind Eng 111(September):492-506

7. Boudella MEA, Sahin E, Dallery Y (2018) Kitting optimisation in Just-in-Time mixed-model assembly lines: assigning parts to pickers in a hybrid robot-operator kitting system. Int J Prod Res 56(16):5475-5494

8. Coelho F, Relvas S, Barbosa-Póvoa AP (2018) Simulation of an order picking system in a manufacturing supermarket using 
collaborative robots. Proceedings 32nd European Conference on Modelling and Simulation, May 22-25, Wilhelmshaven, Germany

9. Boudella MEA, Sahin E, Dallery Y (2016) A mathematical model to assess the performance of a robotic kitting system in an assembly plant. In: proceedings of the 11th International Conference on Modeling, Optimization and Simulation, Aug. Montréal, Canada, pp 22-24

10. Fager P, Calzavara M, Sgarbossa F (2019) Kit preparation with cobot-supported sorting in mixed-model assembly. To be published in the proceedings of $9^{\text {th }}$ IFAC Conference MIM 2019

11. Kootbally Z, Schlenoff C, Antonishek B, Proctor F, Kramer T, Harrison W, Downs A, Gupta S (2018) Enabling robot agility in manufacturing kitting applications. Integr Comput-Aid E 25:193-212

12. Sadrfaridpour B, Wang Y (2018) Collaborative assembly in hybrid manufacturing cells: an integrated framework for human-robot interaction. IEEE T Autom Sci Eng 15(3):1178-1192

13. Neto P, Simão M, Mendes N, Safeea M (2019) Gesture-based human-robot interaction for human assistance in manufacturing. Int J Adv Manuf Technol 101(1-4):119-135

14. Martinez C, Boca R, Zhang B, Chen H, Nidamarthi S (2015) Automated bin picking system for randomly located industrial parts. Proceedings of the 2015 IEEE International Conference on Technologies for Practical Robot Applications (TePRA), May 1115, Woburn, USA

15. Barbazza L, Faccio M, Oscari F, Rosati G (2017) Agility in assembly systems: a comparison model. Assem Autom 37(4):411-421

16. Malik A, Bilberg A (2019) Complexity-based task allocation in human-robot collaborative assembly. Ind Robot 46(4):471-480
17. El Makrini I, Elprama SA, Van den Bergh J, Vanderborght B, Knevels AJ, Jewell CI et al (2018) Working with walt: how a cobot was developed and inserted on an auto assembly line. IEEE Robot Autom Mag 25(2):51-58

18. Gil-Vilda F, Sune A, Yagüe-Fabra JA, Crespo C, Serrano H (2017) Integration of a collaborative robot in a U-shaped production line: a real case study. Procedia Manuf 13:109-115

19. Krüger J, Lien TK, Verl A (2009) Cooperation of human and machines in assembly lines. CIRP Ann 58(2):628-646

20. Wakabayashi Y, Kamioka M, Kinugawa J, Kosuge K (2017) KitPaDy: Robot co-worker in kitting process: basic concept and computation of working time. In: proceedings of the 13th IEEE Conference on Automation Science and Engineering (CASE), Aug, Xi'an, China, pp 20-23

21. Sellers CJ, Nof SY (1989) Performance analysis of robotic kitting systems. Robot Cim-Int Manuf 6(1):15-24

22. Fager P, Hanson R, Medbo L, Johansson MI (2019) Kit preparation for mixed model assembly - efficiency impact of the picking information system. Comput Ind Eng 129(March):169-178

23. Fager P (2018) Kit preparation for mixed-model assembly: efficiency impact of confirmation methods. Ind Manag Data Syst 119(3): $547-560$

24. Battini D, Calzavara M, Persona A, Sgarbossa F (2015) A comparative analysis of different paperless picking systems. Ind Manag Data Syst 115(3):483-503

Publisher's note Springer Nature remains neutral with regard to jurisdictional claims in published maps and institutional affiliations. 